\title{
Synchronous uterine adenocarcinoma and leiomyosarcoma - a case study
}

\author{
Kamila Dudzik ${ }^{1}$, Agnieszka Krzysteczko ${ }^{1}$, Leon Kolny ${ }^{1}$, Agnieszka Bąk ${ }^{1}$, Ewelina Stawicka-Ociepka ${ }^{1}$, \\ Krzysztof Nowosielski ${ }^{2,3}$
}

${ }^{1}$ Department of Gynecology, Obstetrics and Oncological Gynecology, Provincial Specialist Hospital, Tychy, Poland

2Department of Gynecology and Obstetrics, Silesia Institute of Mother and Newborn, Chorzow, Poland

${ }^{3}$ Department of Sexology and Family Planning, Medical College in Sosnowiec, Sosnowiec, Poland

\begin{abstract}
Synchronous gynecological cancers are rarely described. Those cases account for approximately up to $6 \%$ of female genital tract malignancies. The presence of synchronous endometrial adenocarcinoma and gynecological tract neoplasia is rare - the most commonly described is synchronous adenocarcinoma and endometrial ovarian cancer (accounting for $15-20 \%$ of ovarian neoplasia and $5 \%$ of endometrial cancers). Concomitant uterine carcinosarcoma and ovarian cancer, or endometrial adenocarcinoma are extremely rare. Up till now, only 3 cases of synchronous adenocarcinoma and leiomyosarcoma were described.

In the present study a case of 60-year-old woman diagnosed with synchronous endometrial adenocarcinoma and leiomyosarcoma uteri is described. As the preoperative evaluation revealed endometrial adenocarcinoma G2 with intermediate-risk of lymph node metastasis and synchronous leiomyosarcoma G3, total hysterectomy with bilateral salpingo-oophorectomy and systemic lymphadenectomy was performed showing no lymphatic involvement. In the postoperative evaluation the patient was qualified to adenocarcinoma low recurrence-risk group (adenocarcinoma G1 with no LVSI, FIGO IA) - no further radiotherapy was required. However, as synchronous leiomyosarcoma G3 was diagnosed, we decided to refer the patient for adjuvant chemotherapy.

Contemporary recommendation on the diagnosis and treatment of uterine carcinomas, especially uterine leiomyosarcomas, is also described in this paper. The presented case showed that diagnosis and treatment of women with uterine tumors should be individualized as in the same case an extremely rare cancer type can be present which, consequently, changes the treatment regimen and prognosis.
\end{abstract}

Key words: uterus, adenocarcinoma, leiomyosarcoma.

\section{Introduction}

Synchronous gynecological cancers are rarely described. These cases account for approximately up to $6 \%$ of female genital tract malignancies. The most commonly described are synchronous ovarian and endometrial cancers [1-3]. In this study a case of a female patient diagnosed with synchronous uterine leiomyosarcoma and endometrial adenocarcinoma is presented. A scant data based only on a few retrospective studies, histological diversity and vague etiology of synchronous gynecological cancers make it impossible to establish firm diagnostic and treatment recommendations; though each case must be analyzed by a multidisciplinary team of specialists to prepare a therapeutic plan for an individual patient.

\section{Case study}

A 60-year-old woman was admitted with vaginal bleeding to the Department of Gynecology and Gyneco- logical Oncology in Tychy. During the pelvic examination a solid, irregular tumor $14 \times 10 \times 8 \mathrm{~cm}$ in dimension was expelled from the uterus. The material, probably uterine myoma, was sent for histopathological evaluation.

The medical history of the patient was then taken. The woman had no major comorbidities, she was pregnant twice and gave birth to two healthy children. The last menses was when she was 47 years old. Since that time the patient had not visited any gynecological outpatient clinic.

During speculum examination heavy uterine bleeding was noted. No abnormalities in the cervix and vaginal walls were detected. In the bimanual examination, vaginal and uterine prolapse stage II (according to pelvic organ prolapse quantification system - POP-Q II), enlarged, solid corpus of the uterus and normal adnexa was revealed. Bimanual examination revealed vaginal and uterine prolapse stage II (according to pelvic organ prolapse quantification system - POP-Q II), enlarged, solid corpus of the uterus and normal adnexa. The results of transvaginal ultrasound showed retroverted 
uterus, $73 \mathrm{~mm}$ in length and $59 \mathrm{~mm}$ in depth, with regular contour, heterogenous echogenicity with myoma $38 \times 36 \mathrm{~mm}$ and irregular endometrium thickness up to $15 \mathrm{~mm}$. Additionally, subserous myoma $33 \times 24 \mathrm{~mm}$ was noted. The ovaries were not visible.

Since in the standard blood test no abnormalities were detected the patient was qualified for dilation and curettage - the procedure was performed instantaneously due to vaginal bleeding. The next day the patient was discharged from the hospital in good condition.

The histopathological examination revealed:

- Uterine tumor - malignant mixed mesodermal tumor with epithelial tissue (endometrial adenocarcionoma G2) and connective tissue (leiomyosarcoma G3) components.

- Endometrial sampling - endometrial adenocarcinoma G2.

The patient was readmitted to the hospital. Chest $\mathrm{X}$-ray revealed no major abnormalities; abdominal and pelvic computer tomography (CT) showed probable invasion to more than $50 \%$ of myometrium with no suspicious paraaortic and pelvic lymph node (Fig. 1). The oncological multidisciplinary team qualified the patient for the radical surgery. A modified radical hysterectomy with bilateral salpingo-oophorectomy and systemic lymphadenectomy was performed. The histopathological analysis revealed uterine endometrial adenocarcinoma G1 with myometrial invasion less than $50 \%$ and negative paraaortic and pelvic nodes (FIGO IA). Based on both histopathological protocols diagnosis of synchronous endometrial adenocarcinoma G1 FIGO IA (low-risk group) and leiomyosarcoma G3 FIGO IB was made. No further radiotherapy was required. However, due to the presence of leiomyosarcoma G3 the patient was referred for further chemotherapy (gemcitabine/ docetaxel). Currently she tolerates the chemotherapy regiment well - the further prognosis is promising.

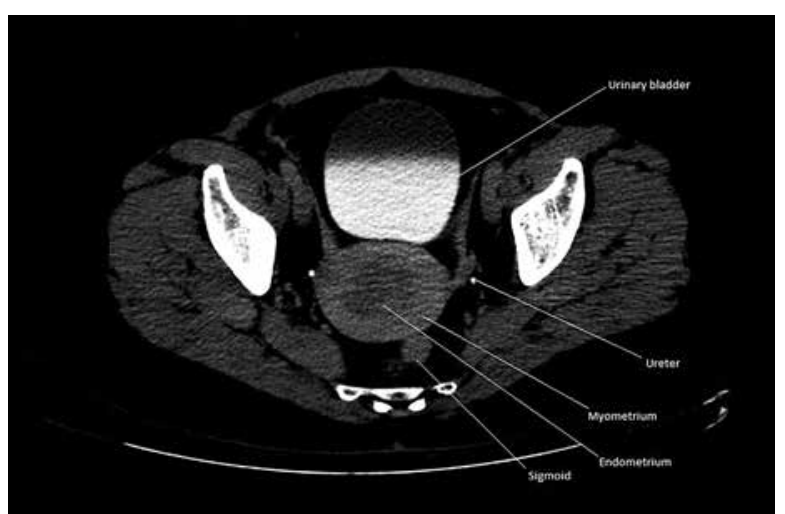

Fig. 1. Preoperative CT scan showing endometrial cancer invading more than $50 \%$ of myometrium with no suspicious paraaortic and pelvic lymph node

\section{Discussion}

Uterine sarcomas are a rare heterogenic group of gynecological malignancies derived from myometrium or endometrial stroma, comprising 3 to $8 \%$ of all uterine cancers [4-7]. According to the National Comprehensive Cancer Network uterine sarcomas are classified to: endometrial stromal sarcoma (21\%), leiomyosarcoma - uLMS (63\%), undifferentiated uterine sarcoma (16\%) $[4,5]$. Treatment of uLMS include total hysterectomy with bilateral salpingo-oophorectomy. The role of systemic lymphadenectomy has not yet been established. Radiotherapy is not recommended, however, adjuvant chemotherapy is believed to be effective for improving overall survival $[4,5]$.

Endometrial adenocarcinomas are one of the most common neoplasia of the female genital tract. Due to the early detection, the prognosis (overall survival, progression-free survival), when proper treatment is introduced, is satisfactory - $79 \%$ of endometrial cancers are detected as stage I with 5-year survival rate up to $90 \%$ $[5,6]$. The treatment of endometrial cancer include total hysterectomy with bilateral salpingo-oophorectomy. Similarly to uterine sarcomas, there is no consensus as to the role of systemic lymphadenectomy, especially in the preoperative suspicion of FIGO stage I. Thus, in the preoperative evaluation, based on endometrial sampling results and abdominal/pelvic CT or magnetic resonance imagining (MRI) scans or uterus transvaginal ultrasound, 3 groups were distinguished: the low risk of lymph node involvement group - grade 1 and 2 , invading less than $50 \%$ of myometrium; intermediate-risk group - grade 1 or 2, invading more than $50 \%$ of myometrium, or grade 3 and invading less than $50 \%$ of myometrium; high-risk group - grade 3 and invading more than $50 \%$ of myometrium. In the case of the low- and intermediate-risk groups the systemic lymphadenectomy is not required; in the high-risk group - it is highly recommended $[5,6,8]$. In the postoperative management adjuvant radiotherapy and chemotherapy is indicated in the high and intermediate recurrence-risk groups (age over 70 and the presence of one risk factor; age over 50 and the presence of two risk factors; any age and the presence of three risk factors). The aforementioned risk factors include: lymphovascular space invasion - LSVI, grade 2 or 3 , invasion to the outer third of myometrium [5-8].

In the presented case, as the preoperative evaluation revealed endometrial adenocarcinoma $G 2$ with intermediate-risk of lymph node metastasis and synchronous leiomyosarcoma G3, the systemic lymphadenectomy was performed revealing no lymphatic involvement. In the postoperative evaluation the patient was qualified to adenocarcinoma low recurrence-risk group (adenocarcinoma G1 with no LVSI, FIGO IA) - no further radiotherapy was required. However, as syn- 
chronous leiomyosarcoma G3 was diagnosed, we decided to refer the patient for adjuvant chemotherapy.

The presence of synchronous endometrial adenocarcinoma and gynecological tract neoplasia is rare the most commonly described is synchronous adenocarcinoma and endometrial ovarian cancer (accounting for $15-20 \%$ of ovarian neoplasia and $5 \%$ of endometrial cancers) [3, 9]. Concomitant uterine carcinosarcoma and ovarian cancer $[10,11]$ or endometrial adenocarcinoma are extremely rare $[12,13]$. Up till now, only 3 cases of synchronous adenocarcinoma and leiomyosarcoma were described [14-16]. In 2000 Sheyn et al. described a case of a 66-year-old woman with that diagnosis [14]. In 2006 Isin Dogan Ekici et al. presented a case of 56-year-old individual with synchronous endometrial adenocarcinoma, leiomyosarcoma and well-differentiated ovarian mucinous cystadenocarcinoma [15]. Crean et al. presented a similar to ours case study in 2016 [16]. As this condition is extremely rare the presented case adds some new information to our knowledge of gynecological malignancies.

In the case of concomitant endometrial cancer with mesenchymal tumor the risk of the presence of carcinosarcoma has to be considered. This is a rare, highly aggressive form of cancer comprising 1 to $3 \%$ of all uterine malignances [4-8, 17]. The differential diagnosis of leiomyoma, leiomyosarcoma and carcinosarcoma based on clinical symptoms or radiological imaging/ultrasonography is difficult; the characteristic radiological and sonographic features of uterine carcinosarcomas have not been established yet [4-8]. They are described as tumors with heterogenous echogenicity similar to typical myomas. Even in MRI the differential criteria are vague [4-8]. In our case there was a spontaneous expulsion of the tumor which definitely facilitated the diagnosis and treatment planning.

\section{Conclusions}

The presented case showed that diagnosis and treatment of women with uterine tumors should be individualized as in the same case an extremely rare cancer type can be present which, consequently, changes the treatment regimen and prognosis.

\section{Disclosure}

Authors report no conflict of interest.

\section{References}

1. Saatli B, Yildirim N, Cenk Ozay A, et al. Synchronous tumors of the female genital tract: A 20-year experience in a single center. Ginekol Pol 2014; 85: 441-445.

2. Tong SY, Lee YS, Park JS, et al. Clinical analysis of synchronous primary neoplasm of the female reproductive tract. Eur J Obstet Gynecol Reprod Biol 2008; 136:78-82.
3. Amalinei C, Balan R, Crauciuc E, Toma O. Synchronous, metachronous and metastatic tumors of the upper female genital tract. Annals of the "Alexandru Ioan Cuza University" Sect. II.a Genetics and Molecular Biology 2008; 9: 1-22

4. NCCN Guidelines. Uterine Neoplasms. Version 1.2017. https://www.nccn. org/professionals/physician_gls/pdf/uterine.pdf (Accessed 05.02.2017).

5. Rutkowski P, Śpiewankiewicz B, Koseła H, et al. Zalecenia postępowania diagnostyczno-terapeutycznego u chorych na mięsaki macicy. Curr Gynecol Oncol 2013; 11: 24-32.

6. Practice Bulletin No. 149: Endometrial cancer. Obstet Gynecol 2015; 125: 1006-1026.

7. Serkies K, Pawłowska E, Jassem J. Postoperative therapy for uterine carcinoma. Overview of randomized trials. Onkol Prakt Klin 2013; 9: 216-224.

8. Kryniewska K, Sznurkowski JJ. Key problems in the treatment of endometrial cancer in the light of ESMO-ESGO-ESTRO Consensus of December 2015. Curr Gynecol Oncol 2016; 14: 145-155.

9. Lee TY, Lee C, Choi WJ, et al. Synchronous occurrence of primary malignant mixed müllerian tumor in ovary and uterus. Obstet Gynecol Sci 2013; 56: 269-272.

10. Chaitra C, Sharma S, Valiathan M, Pai MV. Synchronous occurrence of malignant mixed mulleriantumor in the uterus and ovary: a case report. RJPBCS 2017; 8: 585-591.

11. Karateke A, Akdemir Y, Sozen H, Cakir S. Synchronous Primary Heterorologous Type of Malignant Mixed Mullerian Tumor of the Uterus and Primary Ovarian Cancer. GINECOeu 2011; 7: 62-64.

12. Kuc-Rajca M, Walaszkowska-Czyż A. Mięsakorak trzonu macicy jako trzeci nowotwór pacjentki - opis przypadku i omówienie aktualnych zaleceń postępowania. OncoReview 2013; 3: 274-277.

13. Semczuk A, Colas E, Walczyna B, et al. Coexistence of homologous-type cervical carcinosarcoma with endometrioid-type G1 endometrial cancer: a case report with an immunohistochemical study. Int J Clin Exp Pathol 2014; 7: 7191-7195.

14. Sheyn I, Mira JL, Blanco R, et al. Concomitant well-differentiated adenocarcinoma and leiomyosarcoma of the uterus. Arch Pathol Lab Med 2000; 124: 1539-1541.

15. Işin Doğan Ekici A, Küçükali T, Coşkun Salman M, Ayhan A. Triple simultaneous primary gynecological malignancies in a 56 -year-old patient. Int J Gynecol Cancer 2006; 16: 1947-1950.

16. Crean KK, Huang EC, Alvarez EA. Synchronous uterine adenocarcinoma and leiomyosarcoma: A rare case report causing a clinical conundrum. Int J Surg Case Rep 2016; 22: 32-34.

17. Serkies K, Jassem J. Mięsaki trzonu macicy. Onkol Prakt Klin 2010, 6: 7-13. 